17

1

\title{
Fractional Laser Treatment Boosts Systemic Anti-Tumor Immunity in Combination with OX40 Agonist and PD-1 Blockade
}

\author{
Masayoshi Kawakubo ${ }^{1,2}$ *, Joshua Glahn ${ }^{1,2}$, Shadmehr Demehri ${ }^{1,2}$, Dieter Manstein ${ }^{1,2}$ \\ ${ }^{1}$ Cutaneous Biology Research Center, Department of Dermatology, Massachusetts General Hospital \\ Research Institute, Charlestown, MA 02129, USA \\ ${ }^{2}$ Harvard Medical School, Boston, MA \\ *Correspondence: mkawakubo@mgh.harvard.edu
}

Conflict of interest statement: Patent application number 63/088,281 has been filed (M.K. and D.M.) based on this work ("Intratumoral and Systemic Immunization Using Fractional DamageCreating Device With Checkpoint Molecules for Cancer Therapy”) 


\section{Abstract}

2 While ablative fractional photothermolysis (aFP) with a 10,600 $\mathrm{nm} \mathrm{CO}$ laser is employed for a

3 wide variety of dermatologic conditions, its applications in oncology are relatively unexplored.

4 Building off our previous work, we investigated the effect of unilateral aFP treatment in

5 combination with anti-PD-1 blocking antibody and OX40 agonist on bilateral tumor growth and

6 remission. A CT26 wild type (CT26WT) colon carcinoma cell line was established bilaterally on

7 the hind flanks of a standardized mouse model and tumor characteristics were investigated on

8 aFP treated and untreated sides. Remarkably, triple therapy with fractional $\mathrm{CO}_{2}$ laser in

9 combination with anti-PD-1 antibodies and OX40 agonists resulted in significantly slower tumor

10 growth and complete remissions on bilateral tumors. Flow cytometric analysis showed the triple

11 treatments elicited an increase of granzyme B+CD8+T cells due to synergistic effect of aFP

12 treatment and the checkpoint molecules, including the induction of CD103+ CCR7+ dendritic

13 cells (DCs) in aFP-treated tumor by aFP treatment, XCR1+ DCs in drainage lymph node by anti-

14 PD-1 inhibitor and OX40+ Ki67+ CD8+ T cells in the lymph node by OX40 agonist. Triple

15 therapy-mediated tumor regression and survival was abrogated upon CD8+ T cell depletion.

16 Importantly, when two mismatched cancer cells were implanted into mice, the effect of the triple

17 therapy on distant tumor was abrogated, showing antigen specificity of the T cell immunity

18 induced by triple therapy. This study highlights the efficacy of aFP a novel adjuvant for current

19 cancer immunotherapeutics.

20

21 Keywords 
1 Anti-tumor immunity, Tumor associated antigen, Antigen specific CD8+T cells, Laser therapy,

2 Fractional photothermolysis, Thermal denaturation, Colon carcinoma, CT26WT, PD-1, OX40

3

4

5

6

7

8

9

10

11

12

13

14

15

16 


\section{Introduction}

2 Ablative fractional photothermolysis (aFP) is a laser treatment modality used to generate a

3 regular pattern of microscopic zones of thermal damage in tissue (1). aFP is used for a range of

4 dermatologic conditions including treatment of photodamaged skin, dyschromia, rhytids, and

5 revision of acne, surgical, and burn scars (1-5). The therapy consists of a fractionated pattern of

6 microscopic treatment zones (MTZ) created by focused laser beams at wavelengths highly

7 absorbed by molecules in the tissue. These MTZs consisted of an empty column of ablated tissue

8 surrounded by a ring of denatured collagen and thermally damaged tissue $(6,7)$. MTZ usually

9 measure less than $0.5 \mathrm{~mm}$ across, however, characteristic such as diameter and depth depend on

10 adjustable laser parameters. aFP typically damages only a small fraction of the tissue (often

11 between 5-20\%), leaving the majority of tissue unharmed (8). By creating a path through the

12 stratum corneum, aFP has been used to facilitate transcutaneous drug delivery of

13 chemotherapeutics and photosensitizers in the treatment of cutaneous cancers (9). However, the

14 use of aFP as a primary treatment modality remains poorly understood.

15 In a previous study we investigated the role of aFP in inducing anti-tumor immunity to promote tumor regression both locally and systemically $(10,11)$. We demonstrated that, in combination

17 with an anti-PD-1 inhibitor, aFP promotes the proliferation of tumor antigen specific CD8+ Tcells in both treated and untreated tumors in a bilateral mouse tumor model. While treatment led to near complete remission in irradiated tumors, aFP+ anti-PD-1 inhibitor therapy only resulted

20 in contralateral tumor size and growth rate reduction in $33 \%$ of mice (11). We theorize that the

21 limited success of systemic anti-tumor immunity despite bilateral infiltration of the CD8+ T-cells

22 is the ratio of regulatory $\mathrm{T}$-cells $\left(\mathrm{T}_{\text {reg }}\right)$ to $\mathrm{CD} 8+\mathrm{T}$-cells in the contralateral tumor, blocking the antigen-specific immunity promoted by aFP. 
1 To overcome this proposed immune blockade, an OX40 agonist was added to aFP + anti-PD-1

2 inhibitor therapy. OX40 agonists are shown to promote the activation, proliferation and effector

3 function of killer CD8+ T-cells (12-14) in addition to promoting the proliferation and effector

4 function of helper CD4+ T-cells (15-17) and depleting the number of $\mathrm{T}_{\text {reg }}$ cells (18-21). By

5 repressing the regulatory checkpoints inhibiting CD8+ T-cell effects systemically, triple therapy

6 with aFP+ anti-PD-1 inhibitor + OX40 agonist has the potential to become a novel

7 immunomodulatory therapy for promoting tumor regression both at the treatment site and in

8 remote tumors not treated with aFP.

\section{Results}

\section{aFP treatment boosts effect of systemic anti-tumor immunity induced by anti-PD-1} inhibitor and OX40 agonist combination therapy.

13 We established a two-tumor mouse model, with identical tumors seeded on each hind leg, to evaluate the effect of aFP treatment in conjunction with immune checkpoint molecules on systemic induction of anti-tumor immunity. In the initial experiment, tumors were left for 6 days post inoculation, reaching an average diameter of $4 \mathrm{~mm}$, before aFP treatment. Intraperitoneal

17 treatment with anti-PD-1 inhibitor and/or OX40 agonist occurred on days 6, 8, 10, 12, and 14 after inoculation. Triple therapy with aFP + anti-PD-1 + OX40 agonist led to a significant reduction in tumor volume and growth rate following treatment (Figure 1a and b). Triple therapy resulted in complete tumor remission in all mice with uniform survival past the 90-day

21 predetermined endpoint (Figure 1c). Significance value between survival curves are shown in

22 Table 1. Of note, there was no significant difference between triple therapy and therapy with 
1 therapy, a second round of experiments was conducted with treatment beginning 12 days after

2 inoculation and an average tumor diameter of $7 \mathrm{~mm}$. aFP treatment was performed on day 12 and

3 anti-PD-1 inhibitor and/or OX40 agonist were administered on days 12, 14, 16, 18, and 20 after

4 inoculation. Triple therapy in the delayed treatment group led to a significant reduction in tumor

5 volume and growth rate over therapy with anti-PD-1 inhibitor and OX40 agonist (Figure 2a, b

6 and c). Both aFP treated and contralateral tumors achieved complete remission in the triple

7 therapy group and 8 of 10 mice survive to 90 days compared to tumor shrinkage in 3 of 10 mice

8 in the double therapy group (Figure $2 \mathrm{~d}$ ). Significance values for differences between survival

9 curves are shown in Table 2.

11 Surviving mice develop long-term anti-tumor immunity

12 To evaluate for long-term induction of anti-tumor immunity, mice from the double and triple

13 therapy groups that survived 90 days post original inoculation with complete tumor remission

14 and a group of age-matched tumor naive mice received a subcutaneous inoculation of $3.5 \times 10^{5}$

15 CT26WT cells in right thigh (aFP-untreated thigh in case of aFP-treated mice). While tumors on

16 the tumor-naive control mice progressed over time leading to no survivors at 30 days, tumors in

17 the rechallenged double and triple therapy groups showed no signs of tumor progression and

18 remained tumor-free for at least 60 days post inoculation (Figure 3). Significance values

19 between the survival curves are: control vs. anti-PD-1 + anti-OX40 $(p<0.0001)$, control vs. aFP + 20 anti-PD-1 + anti-OX40 $(p<0.0001)$. 
1 To investigate the mechanism by which triple therapy led to regression in untreated contralateral

2 tumors, flow cytometry of untreated tumor cells 5 days post-aFP were analyzed for the

3 concentration of tumor-infiltrating CD3+, CD4+, CD8+, and granzyme-B+ CD8+ T-cells, along

4 with Foxp3+ regulatory $\mathrm{T}$-cells $\left(\mathrm{T}_{\text {regs }}\right) . \mathrm{CD} 8+$ and granzyme $\mathrm{B}+\mathrm{CD} 8+\mathrm{T}$-cells significantly

5 increased by tumor weight in the triple therapy group compared to all other treatment groups

6 (CD8/weight: control, anti-PD-1, anti-OX40, aFP, aFP + anti-PD-1 or aFP + anti-OX40 vs. aFP

$7+$ anti-PD-1 + anti-OX40 ( $p<0.05$; Figure 4d-e). However, there was no significant difference

8 in CD3+ and CD4+ T-cell concentrations between experimental groups (Figure 4a-b).

9 Furthermore, there was no significant difference between number of $T_{\text {reg }}$ cells between groups

10 (Figure 4c), resulting in an increased ratio of CD8+ T-cells to $\mathrm{T}_{\text {reg }}$ in triple therapy (control, anti-

11 PD-1, or aFP + anti-PD-1 vs. aFP + anti-PD-1 + anti-OX40 ( $p<0.05$; Figure 4f).

\section{aFP + anti-PD-1 therapy increases CD103+ CCR7+ dendritic cells in aFP-treated tumor} and XCR1+ DCs in drainage lymph node

To investigate how triple therapy significantly increases tumor-infiltrating CD8+ T lymphocyte migration into contralateral tumors, we measured the number of CD45+ CD11c+ $\mathrm{MHC}$ II + dendritic cells (DCs) present in in aFP-treated tumor and associated drainage lymph nodes 3 days post-treatment using flow cytometry. For this subset of experiments, we did not used OX40 agonists due to the lack of OX40 receptors on target DCs. Initially, we measured the concentration of CD103+ CCR7+ dendritic cells in aFP-treated and control tumors. CD103+ DCs were chosen as the specific subpopulation with the ability to induce proliferation of naïve CD8 T-cells (22) in the presence of antigen, and migration of DC to local lymph nodes is directly mediated by the CCR7 chemokine receptor (responsive to chemoattractant CCL19 and CCL21) 
1 (24). We found an increase in the concentration of CD103+ CCR7+ DCs normalized to tumor

2 weight in aFP treatment groups (aFP and aFP + anti-PD1) when compared to control and anti-

3 PD1 only groups (Figure 5a), with the aFP + anti-PD-1 group showing a significant increase in

4 DC concentration over anti-PD-1 alone $(p<0.05)$.

5 Next, we measured the number of DCs expressing XCR1 in drainage lymph node. The

6 interaction between naive CD8+ T-cells and cross-presenting DCs is mediated by the XCR1-

$7 \mathrm{XCL1}$ axis. XCL1 is a chemoattractant secreted by CD8+ T-cells and is sensed by XCR1 ligands

8 on DCs. This interaction enhances cross-priming, making the XCR1-XCL1 axis an integral

9 component in the development of efficient cytotoxic immunity $(25,26)$. As shown in Figure 5b,

$10 \mathrm{XCR} 1+\mathrm{DC}$ concentrations increased significantly in the aFP + anti-PD-1 group as compared to

11 control and aFP only groups $(p<0.05)$. Taken together, these data suggest that aFP treatment

12 recruits DCs with the capability of inducing proliferation of naive CD8+ T-cells, enabling their

13 migration to drainage lymph node on the aFP-treated tumor side, and anti-PD-1 inhibitor

14 increases DCs and is an integral component in the development of efficient cytotoxic immunity.

\section{Triple therapy induces expression of $\mathrm{OX} 40$ and PD-1 on CD8+ T cells in local drainage}

\section{7 lymph nodes}

18 To investigate why triple therapy resulted in higher concentrations of tumor-infiltrating CD8+ T

19 lymphocytes in the contralateral, untreated tumor, we quantified expression of PD-1, OX40, and

$20 \mathrm{Ki} 67$ on CD8+ T-cells in local lymph nodes 5 days after aFP treatment. OX40 and PD-1 are

21 expressed rapidly after antigen stimulation on CD8+ T-cells $(27,28)$, meaning the expression of

22 OX40 and PD-1 is a marker of stimulation. 
1 Flow cytometry confirmed the expression of OX40+ PD-1+ CD8+ T-cells in drainage lymph nodes on the aFP-treated side with groups receiving triple therapy displaying significantly higher number of T-cells. The basis of immunomodulation in triple therapy is the ability of OX40 agonists to promote proliferation of OX40-expressing T-cells while the anti-PD-1 antibodies counteract the immune suppression caused by the PD-1/PD-L1 pathway. Significantly increased proliferation of OX40+CD8+ T-cells in the triple therapy treatment group was confirmed by staining for Ki67, a common marker of proliferation. The experimental groups receiving aFP, OX40 agonists, and PD-1 antagonist (triple therapy) demonstrated significantly higher proportion of Ki67+ staining cells than OX40 agonist alone group $(p<0.01)$ and than control and anti-PD-1 groups $(p<0.05$; Figure 6a). Moreover, the increase in PD-1+ OX40+ Ki67+ CD8+ T-cells in the triple therapy group increased significantly when compared with any other experimental group except $\mathrm{FP}+\mathrm{OX} 40$ group, indicating that triple therapy is an effective promoter of antigen-stimulated CD8+ T-cell proliferation, leading to higher systemic concentrations of immune cells and infiltration into contralateral tumors (control, anti-PD-1, anti-OX40, or aFP vs. triple therapy ( $p<0.005)$, anti-PD-1 + anti-OX40 or aFP + anti-PD-1 vs. aFP + anti-PD-1 + anti-OX40 ( $p<0.05)$; Fig. 6b).

\section{Adaptive immunity is crucial to eradicating cancer cells after the triple therapy}

To confirm the role of adaptive immunity in eradicating cancer cells systemically with triple therapy, both groups received triple therapy and the experimental group received anti-CD8 depletion antibodies to eliminate CD8+ T-cell populations in treated mice. The beneficial effects of triple therapy were counteracted by suppression of CD8+ T-cell function, with the group receiving anti-CD8+ depletion antibodies showing no survival past 30 days and 4 out of 5 mice 
1 in the triple therapy control group surviving the full 90 days $(p=0.0018$; Figure $7 \mathrm{a}-\mathrm{b})$. These

2 results indicate that $\mathrm{CD} 8+\mathrm{T}$-cells and adaptive immunity play a critical role in the therapeutic

3 benefits of triple therapy.

4

Antigen specificity is necessary for systemic anti-tumor immunity induced by aFP

To demonstrate the antigen specificity of triple therapy on distant tumors, we developed a mismatched mouse model by inoculating CT26WT tumor cells in the left leg and 4T1 tumor cells (murine mammary carcinoma) in the right leg. This experimental group was compared with a matched mouse model with inoculation of CT26WT tumor cells in both left and right legs. Triple therapy with aFP was performed on the left haunch only. While the matched mouse group saw complete remission of both tumors and survival of all mice past 90 days, the mismatched mouse group saw complete remission of the aFP treated tumor without a comparable effect on the mismatched $4 \mathrm{~T} 1$ side, resulting in no survival past 30 days $(p=0.0035$; Figure $8 \mathrm{a}$ and $\mathrm{b})$. The benefits did not extend to the contralateral side, suggesting an antigen specific mediated reaction to aFP.

\section{aFP treatment induces expression of HSP70, -90, and calreticulin on tumor cells}

There is evidence that extracellular localized and membrane-bound heat shock proteins (HSPs) play a key role in eliciting antitumor immune responses by acting as carriers for tumor-derived immunogenic peptides, enhancing presentation of antigens as targets for the innate immune system (29). Additionally, the expression of calreticulin (CALR), the so called "eat me sign", has been associated with enhanced immune function and shares a common receptor (LRP1) with 
1 HSP70 and -90 on CD103+ dendritic cells (30). To investigate the effect of aFP on transcription

2 of HSP70 and -90 precursors, RNA sequencing was performed 24-hours after tumor irradiation.

3 Both HSPala and HSPa1b, mRNA precursors of HSP70, were found to be upregulated in aFP

4 treated tumors as compared to control (Supplemental Table 1). To measure the expression of

5 HSP70, -90, and calreticulin in aFP treated tumor cells, CT26WT colon carcinoma cells were

6 transfected with green fluorescence protein (GFP) for detection. On day 3 after irradiation, flow

7 cytometry confirmed that expression of HSP70, -90, and CALR on GFP transfected cells was

8 significantly higher than in the control group (Figure 9a-c). Furthermore, the proportion of

9 CD103+ DCs expressing LRP1 by tumor weight was significantly higher in the aFP-treated

10 tumor (Figure 9d). The increase in HSP70, -90, and CALR, along with the augmented

11 concentration of their receptor, suggests aFP itself induces expression of molecules which

12 potentially promote anti-tumor immunity.

\section{Discussion}

15 This study illustrates the benefits of triple therapy with ablative fractional photothermolysis (aFP), anti-PD-1 antibodies, and OX40 agonists in initiating a systemic, tumor specific immune

17 response leading to a significant reduction of tumor burden. While aFP is currently used for a

18 variety of clinical indication, its therapeutic value is generally thought of in terms of localized

19 tissue effects within a limited treatment area for the stimulation of wound healing or tissue

20 regeneration. This study builds on our prior work, expanding the applications of aFP into the

21 world of immunotherapeutics $(10,11)$. Fractional laser therapy's ability to induce tumor

22 regression relates to tumor associated antigen (TAA). Although Mroz et al demonstrated the 
1 tumor cells at baseline is not sufficient to stimulate an immune response to prevent tumor growth

$2(31,32)$. The basis of aFP treatment is the release of TAA through thermal denaturation of tumor

3 cells, potentially enhancing anti-tumor immunity by allowing cytotoxic T-cells to recognize and

4 bind the immunodominant peptide epitope $(10,11)$. The choice of a fractionated laser pattern of

5 approximately 5\% microscopic treatment zone (MTZ) to tumor ratio was targeted at enhancing

6 inflammation while avoiding direct killing of the local tumor (Supplement figure1) (10, 11). In

7 our prior study, we found that aFP treatment of CT26WT tumors both induced anti-tumor

8 immunity and improved long term survival (11). Huang et al demonstrated that the CT26WT

9 colon carcinoma cells line have a dominant TAA in a single peptide known as AH-1, a nonmutated nonamer derived from the envelope protein ( $\mathrm{gp} 70)$ of an endogenous ecotropic murine

11 leukemia provirus (33). Pentamer staining in our previous study revealed an increase in AH1

12 specific CD8+ T-cells normalized to tumor weight in aFP-treated groups, supporting the theory

13 that aFP promotes the release of TAA and induces anti-tumor immunity (11). Our current study

14 further substantiates this claim by observing an increase in CD103+ CCR7+ DCs in aFP-treated

15 tumors consistent with the increase in local TAA and the generation of damage-associated

16 molecular patterns (DAMPs) (34). The choice of ideal laser dosimetry, including variation in

17 pulse energy, ablative wavelength, and pattern density to maximize thermal damage and the

18 release of DAMPs deserves further study. The second component of triple therapy, anti-PD-1

19 inhibitory antibodies, also contributes to anti-tumor immunity. PD-1 is a cell surface protein used

20 to dampen the immune response and is observed in multiple subset of activated lymphocytes,

21 including T- (35), B- (36), and NK-cells (37). PD-1 expression on CD8+ T cells is primarily due

22 to antigen-driven T-cell receptor (TCR) signaling, with expression of the protein within 2-4

23 hours after antigen exposure and before cell division (27). PD-L1 and PD-L2 are co-inhibitory 
1 factors of the immune response that bind to cells expressing PD-1 to decrease their capacity for

2 cytotoxic response. PD-L1 is normally expressed on dendritic cell (DC) wherein interactions

3 between DC and T-cell via the PD1/ PD-L1 axis suppresses proliferation and potentiates

4 apoptosis of T-cell $(38,39)$. Cancer cells can also express PD-L1 (40) to evade the cytotoxic

5 effect of CD8+ T cell by suppressing cell proliferation through the PD-1/ PD-L1 axis (35, 41,

6 42). Our study employs anti-PD-1 antibodies to inhibit the suppressor effect of PD-1 expression

7 by cancer cells. Consistent with this reasoning, there are reportes that blocking the PD-1/PD-L1

8 axis lead to increased expression of granzyme B in CD8+ T-cells in the presence of antigen

9 along with increased proliferation and anti-tumor function $(27,39)$. In our previous study, we

10 reported the synergistic effect of aFP and anti-PD-1 inhibitors leading to the increase of TAA-

11 specific CD8+ T-cells systemically (11). In our present study, we confirmed that aFP + anti-PD-

121 therapy led to an increase in XCR1+ DC in drainage lymph nodes, further validating the

13 mechanism by which the two interventions augment anti-cancer immunity. However, despite our

14 observation of TAA-specific CD8+ T-cells in contralateral, untreated tumors with aFP + anti-

15 PD-1 therapy, only 33\% of untreated contralateral tumors achieved complete remission (11). We

16 posited that the greater effect on local tumors over the abscopal effect on remote cancer cells was

17 due to the higher ratio of $\mathrm{CD} 8+\mathrm{T}$-cells compared to the baseline $\mathrm{T}_{\text {reg }}$ cell population in the local

18 tumor since the ratio in the untreated contralateral tumors was low and there was no significant

19 difference compared to the no treatment control group.

20 To address this, we added a booster of the immune checkpoint agonist OX40 to increase the

21 concentration of CD8+ T-cell systemically. OX40 is a secondary co-stimulatory immune

22 checkpoint molecule, predominantly expressed by T-cells during antigen-specific priming in the

23 presence of inflammatory cytokines such as Interleukin-1 (Il-1) (28). OX40's ligand, known as 
OX40L or CD252, is expressed on activated professional antigen-presenting cells like dendritic cells (DCs), macrophages, and B cells $(43,44)$. Costimulatory signals via the OX40/OX40L axis promotes T-cell expansion, proliferation (12-14), and enhances the activation and effector function of killer T- and helper T-cells (15-17). Furthermore, there is some evidence that OX40 agonists may also be implicated in depleting the number of Foxp3 $+\mathrm{T}_{\text {reg }}$ cells, further contributing to the increased CD8+ T-cell to $\mathrm{T}_{\text {reg }}$ ratio (18-21). In this study, although $\mathrm{T}_{\text {reg }}$ depletion was not observed, triple therapy with aFP + anti-PD-1 + OX40 agonist led to a significant increase in CD8+ T-cells and complete remission was observed in both local and untreated contralateral tumors when treatment was started 6 days following tumor inoculation.

We propose the following mechanism for the increased efficacy of triple. 1) aFP-induced thermal denaturation of tissue releases TAA (11) and DAMPs (34), along with chemokines and cytokines, upregulating the expression of HSP70, HSP90, CALR and increasing expression of antigen on cell surfaces. 2) The chemokines, cytokines, and DAMPs recruit innate immune cells such as DCs $(45,46)$ which present TAA to T-cells via the interaction of MHC and TCR. In the presence of Il-1, TCR signaling stimulates the expression of OX40 on T-cells (Supplemental table 1)(28). 3) The presence of OX40 agonist induces T-cell proliferation, activation, and effector function leading to an increased number of granzyme B+CD8+ T-cells. 4) The addition of PD-1 inhibitors blocks the PD-1/PD-L1 axis that would otherwise inactivate CD8+ T-cells and inhibit the immune response. Taken together, triple therapy works to enhance the immune response to antigen-bearing tumor cells, increasing the effector CD8+ T-cells systemically, leading to the effective eradication of cancer. In support of this mechanism, neither aFP + OX40 agonist nor aFP + anti-PD-1 were sufficient relatively to create a systemic immune response leading to effective tumor regression. In the aFP + OX40 agonist group, tumors could not be 
1 cured effectively and CD8+ T-cells did not infiltrate by 5 days after treatment started. There are

2 reports that PD-1 is expressed on innate cells such as DCs and macrophage (38), and expression

3 of PD-L1 by tumor cells is induced by exposure to Type I and II interferons (35, 47-49), and PD-

4 L1 can even be secreted by tumor cells (50-52). Moreover, activating CD8+ T cells in tumors is

5 reduced in the absence of blocking the PD-1/ PD-L1 axis (39). In our study, expression of PD-L1

6 on tumor cells and induction of interferon beta one at the RNA level in peripheral tumor

7 microenvironment were observed after aFP treatment (Supplement figure 2 and table 1).

8 Therefore, we expect high PD-L1 expression on tumor cells in the presence of interferon beta

9 induced by aFP, secretion of PD-L1 from tumor cells and the suppressive state of innate

10 immunity at peripheral tumor microenvironment in the absence of PD-1 inhibitors leading to

11 fewer DC, the interaction of MHC/ TCR between the innate and adaptive immune cells, and the

12 infiltration of CD8+ T cells into tumors, leading to fewer cured mice than in triple therapy.

13 Similarly, in the aFP + PD-1 group, while we did see an increase in DCs over control, the

14 population of CD8+ T-cells was unable to expand sufficiently in the absence of OX40 agonist to

15 achieve the same robust increase in survival as triple therapy. Interestingly, therapy with anti-

16 PD-1+ OX40 agonist alone led to complete tumor remission in $83 \%$ of mice compared to $100 \%$

17 in the triple therapy group when treatment was initiated 6 days after tumor inoculation. However,

18 tumor volume curves show that the tumor volume peak in cured mice occurs two days later in

19 the anti-PD-1+ OX40 agonist group when compared to the triple therapy group, indicating that

20 the tumor shrank at a slower rate. We posit that the baseline excretion of TAA and expression of

21 HSP70, HSP90 and CALR on tumor cells, plus the low expression of OX40 on T-cells in the

22 absence of inflammatory cytokines, were insufficient to induce anti-tumor immunity. This is

23 consistent with the flow cytometric data showing that total CD8+ T-cell populations in lymph 
1 nodes and tumors in the anti-PD-1+ OX40 agonist alone group were not increased compared to

2 control 5 days after treatment. When treatment was started 12 days after tumor inoculation, only

$330 \%$ of mice receiving anti-PD- $1+$ OX40 agonist alone were cured compared to $80 \%$ in the triple

4 therapy group. We posit that the significant difference between experimental groups relates to

5 the rate at which CD8+ T-cells proliferated. It is generally thought that initiating an immune

6 response against larger tumors is difficult because they grow quickly and implement immune

7 invasion techniques like recruiting $\mathrm{T}_{\text {reg }}$ and myeloid derived suppressor cells (MDSC), secreting

8 immune suppressor cytokines, and down-regulating MHC class I molecules to reduce the

9 expression of immunogenic antigens $(53,54)$. By delaying the onset of treatment, the

10 proliferation of CD8+ T-cells in the anti-PD-1+ OX40 agonist alone group was not sufficient to

11 produce a high enough concentration of T-cells to cure a rapid growing tumor. In terms of

12 monotherapy with anti-PD-1 or OX40 agonist, neither increased the CD8+ or granzyme B+

$13 \mathrm{CD} 8+\mathrm{T}$-cell populations due to the absence of anti-PD-1 inhibitor or OX40 agonist, whose

14 mechanism is mentioned above, resulting in poor survival outcomes.

15 In conclusion, the addition of an OX40 agonist to aFP and an anti-PD-1inhibitor effectively

16 boosted systemic immunity. These effects are likely mediated by an immune cascade including

17 the release of TAA by aFP treatment, increased proliferation of TAA specific CD8+ T cells

18 induced by OX40 agonist, and inhibition of the immune suppressing PD-1/PD-L1 axis by anti-

19 PD-1 inhibitor.

20

21 Materials and Methods

22 Cell lines 
1 CT26WT murine colon and 4T1 murine mammary carcinoma cell lines (ATCC, Mannassas, VA)

2 were cultured in RPMI Medium supplemented with 10\% heat-inactivated fetal bovine serum,

3 penicillin $(100 \mathrm{U} / \mathrm{mL})$, and streptomycin $(100 \mathrm{mg} / \mathrm{mL})($ Sigma-Aldrich, Natick, $\mathrm{MA})$ at $37^{\circ} \mathrm{C}$ in

$45 \% \mathrm{CO}_{2}$. Cultures were performed in $75 \mathrm{~cm}^{2}$ flasks (Falcon, Invitrogen, Carlsbad, CA).

\section{Animals}

6 Six-week-old female BALB/c mice (Charles River Laboratories, Boston, MA) were used for the

7 study. The care and handling of the animals were done in accordance with a protocol approved

8 by the Subcommittee on Research Animal Care (IACUC) at Massachusetts General Hospital

$9(\mathrm{MGH})$.

\section{Animal tumor model}

11 In the primary experiment, mice were anesthetized by intraperitoneal injection of a ketamine (90

$12 \mathrm{mg} / \mathrm{kg})$ and xylazine $(10 \mathrm{mg} / \mathrm{kg})$ cocktail before depilation of bilateral external thighs and

13 subcutaneous inoculation with $3.5 \times 10^{5}$ CT26WT cells. Anti-PD-1 blocking and/or anti-OX40

14 agonist antibodies (29F.1A12 and OX-86 respectively; BioXCell,West Lebanon, NH) were

15 administered intraperitoneally at doses of $200 \mu \mathrm{g}$ per mouse on days $6,8,10,12$, and 14 in the 6

16 day inoculation protocol and on days 12, 14, 16, 18 and 20 after tumor cell inoculation in the 12

17 day protocol (Figure 10). Tumor volume was determined at least 2 times per week by measuring

18 the longest dimension and orthogonal dimension of the tumor with vernier calipers. Tumor

19 volumes were calculated according to the formula volume $=4 \pi / 3 \times[(a+b) / 4]^{3}$, where $a$ and $b$

20 represent the long and short axis lengths, respectively. If tumor volume exceeded $500 \mathrm{~mm}^{3}$ or

21 showed severe ulceration, mice were removed from the study and defined as having reached

22 their endpoint. In the T-cell depletion experiment, anti-CD8+ depletion antibodies (2.43; 
1 BioXCell,West Lebanon, NH) were administered intraperitoneally at a dose of $200 \mu \mathrm{g}$ per mouse

2 every 3 days from one day before tumor inoculation to removal of mice as an endpoint.

3 Fractional $\mathrm{CO}_{2}$ laser irradiation

4 Ablative fractional laser treatment was performed on the tumor site on days 6 or 12 after tumor

5 cell inoculation (Figure 10). 6 days post inoculation tumor size averaged $4 \mathrm{~mm}$ and measured an

6 average of $7 \mathrm{~mm}$ at day 12. Exposures were performed on day 6 or day 12 with an Ultrapulse

7 Encore $\mathrm{CO}_{2}$ laser (Lumenis Inc, Yokneam, Israel). A single pass was performed within a

8 treatment area of $5 \times 5 \mathrm{~mm}$, with a pulse energy of $100 \mathrm{~mJ}, 5 \%$ density, and $300 \mathrm{~Hz}$ frequency.

9 No skin cooling was applied and the anesthesia was performed by intraperitoneal injection of a

10 ketamine $(90 \mathrm{mg} / \mathrm{kg})$ and xylazine $(10 \mathrm{mg} / \mathrm{kg})$ cocktail.

\section{Rechallenge}

12 Mice surviving 90 days after tumor inoculation were rechallenged with matched tumor cells (3.5

$13 \times 10^{5}$ CT26WT) on the contralateral (right) leg from the previously aFP-treated side. Age-

14 matched naive mice were inoculated with the same number of the cells in the right leg as the

15 controls. Inoculated mice were monitored for another 60 days to confirm tumorigenesis.

\section{Flow cytometry analysis}

17 Flow cytometry was used to analyze changes in CD3+ and CD8+ lymphocytes, regulatory $\mathrm{T}$ cell

18 ( $\left.\mathrm{T}_{\text {reg}}\right)$, and dendritic cell (DCs) populations in treatment and control groups. Freshly excised

19 CT26WT tumors were mechanically dissociated through a $70 \mu \mathrm{m}$ strainer into 6-well culture

20 plates containing DNaseI (10 $\mu \mathrm{g} / \mathrm{ml}$, Roche; Nutley, NJ) and collagenase $(10 \mathrm{mg} / \mathrm{ml}$, Life

21 technologies) and incubated for 60 minutes at $37^{\circ} \mathrm{C}$. Dissociated cells were blocked using anti-

$22 \mathrm{CD} 16 / \mathrm{CD} 32$ antibody (93) for 15 minutes at $4^{\circ} \mathrm{C}$ before being stained with anti-CD45 (30-F11),

23 -CD3 (145-2C11), -CD4 (RM4-5), -CD8a (53-6.7), -CD103 (2E7), -OX40 (OX-86; all from 
1 eBioscience; Santa Clare, CA), -CD11c (HL3; BD Biosciences; San Jose, CA), -IA/IE

2 (M5/11415.2), -CCR7 (4B12), or -XCR1 (ZET; all from BioLegend; San Diego; CA) antibodies

3 for 30 minutes at $4^{\circ} \mathrm{C}$. Following staining for surface markers, cells were fixed and

4 permeabilized for 30 minutes at room temperature using the Foxp3/Transcription Factor Staining

5 Buffer Set (eBioscience) according to the manufacturer's instructions. Permeabilized cells were

6 incubated overnight at $4^{\circ} \mathrm{C}$ with anti-Foxp3 (FJK-16s), -Ki67(SolA15), or -granzyme-B (NGZB;

7 all from eBioscience) antibodies. Flow cytometry was performed the day after staining with a

8 Fortessa X-20 (BD Biosciences).

9 Statistics

10 All experiments were repeated at least once. All statics analyses were performed with GraphPad

11 Prism 9.0 (GraphPad Software). All values are expressed as the mean $\pm \mathrm{SD}$. Flow cytometric

12 results were compared with one-way ANOVA. Survival analysis was performed using the

13 Kaplan-Meier method and a log-rank test. Values of $\mathrm{P}<0.05$ were considered statistically

14 significant.

\section{Acknowledgments}

18 The authors wish to thank Lumenis for providing the CO2 laser as a gift, and Tuanlian Luo for preparing $\mathrm{H} \&$ E stained slides.

\section{Author contribution}

22 M.K. contributed to the conception, and design of the work and the acquisition,

23 analysis, and interpretation of data; J.G. contributed to the acquisition of data and the

24 proofreading of the manuscript; S.D. contributed to the interpretation of data; D.M. contributed

25 to the conception of the work. 


\section{Additional Information}

Competing financial interests: Patent application number 63/088,281 has been filed (M.K. and D.M.) based on this work ("Intratumoral and Systemic Immunization Using Fractional DamageCreating Device With Checkpoint Molecules for Cancer Therapy”)

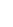

\section{References}

1. Manstein D, Herron GS, Sink RK, Tanner H, Anderson RR. Fractional photothermolysis: a new concept for cutaneous remodeling using microscopic patterns of thermal injury. Lasers Surg Med. 2004;34(5):426-38.

2. Tierney EP, Hanke CW, Petersen J. Ablative fractionated CO2 laser treatment of photoaging: a clinical and histologic study. Dermatol Surg. 2012;38(11):1777-89.

3. Prignano F, Campolmi P, Bonan P, Ricceri F, Cannarozzo G, Troiano M, et al. Fractional CO2 laser: a novel therapeutic device upon photobiomodulation of tissue remodeling and cytokine pathway of tissue repair. Dermatol Ther. 2009;22 Suppl 1:S8-15.

4. Kim HW, Chang SE, Kim JE, Ko JY, Ro YS. The safe delivery of fractional ablative carbon dioxide laser treatment for acne scars in Asian patients receiving oral isotretinoin. Dermatol Surg. 2014;40(12):1361-6.

5. Trelles MA, Velez M, Gold MH. The treatment of melasma with topical creams alone, $\mathrm{CO} 2$ fractional ablative resurfacing alone, or a combination of the two: a comparative study. J Drugs Dermatol. 2010;9(4):315-22.

6. Khan MH, Sink RK, Manstein D, Eimerl D, Anderson RR. Intradermally focused infrared laser pulses: thermal effects at defined tissue depths. Lasers Surg Med. 2005;36(4):270-80.

7. Hantash BM, Bedi VP, Chan KF, Zachary CB. Ex vivo histological characterization of a novel ablative fractional resurfacing device. Lasers Surg Med. 2007;39(2):87-95.

8. Tierney EP, Kouba DJ, Hanke CW. Review of fractional photothermolysis: treatment indications and efficacy. Dermatol Surg. 2009;35(10):1445-61.

9. Wenande E, Erlendsson AM, Haedersdal M. Opportunities for laser-assisted drug delivery in the treatment of cutaneous disorders. Semin Cutan Med Surg. 2017;36(4):192-201.

10. Kawakubo M, Demehri S, Manstein D. Fractional laser exposure induces neutrophil infiltration (N1 phenotype) into the tumor and stimulates systemic anti-tumor immune response. PLoS One. 2017;12(9):e0184852.

11. Kawakubo M, Cunningham TJ, Demehri S, Manstein D. Fractional Laser Releases Tumor-Associated Antigens in Poorly Immunogenic Tumor and Induces Systemic Immunity. Sci Rep. 2017;7(1):12751.

12. Song J, So T, Cheng $M$, Tang $X$, Croft $M$. Sustained survivin expression from OX40 costimulatory signals drives T cell clonal expansion. Immunity. 2005;22(5):621-31.

13. Weinberg AD, Rivera MM, Prell R, Morris A, Ramstad T, Vetto JT, et al. Engagement of the OX-40 receptor in vivo enhances antitumor immunity. J Immunol. 2000;164(4):2160-9.

14. Biagi E, Dotti G, Yvon E, Lee E, Pule M, Vigouroux S, et al. Molecular transfer of CD40 and OX40 ligands to leukemic human B cells induces expansion of autologous tumor-reactive cytotoxic T lymphocytes. Blood. 2005;105(6):2436-42.

15. Gramaglia I, Weinberg AD, Lemon M, Croft M. Ox-40 ligand: a potent costimulatory molecule for sustaining primary CD4 T cell responses. J Immunol. 1998;161(12):6510-7.

16. Dannull J, Nair S, Su Z, Boczkowski D, DeBeck C, Yang B, et al. Enhancing the immunostimulatory function of dendritic cells by transfection with mRNA encoding OX40 ligand. Blood. 2005;105(8):3206-13.

17. Murata S, Ladle BH, Kim PS, Lutz ER, Wolpoe ME, Ivie SE, et al. OX40 costimulation synergizes with GMCSF whole-cell vaccination to overcome established CD8+ T cell tolerance to an endogenous tumor antigen. J Immunol. 2006;176(2):974-83.

18. Kitamura N, Murata S, Ueki T, Mekata E, Reilly RT, Jaffee EM, et al. OX40 costimulation can abrogate Foxp3+ regulatory T cell-mediated suppression of antitumor immunity. Int J Cancer. 2009;125(3):630-8. 
19. Piconese $\mathrm{S}$, Valzasina B, Colombo MP. OX40 triggering blocks suppression by regulatory $\mathrm{T}$ cells and facilitates tumor rejection. J Exp Med. 2008;205(4):825-39.

20. Valzasina B, Guiducci C, Dislich H, Killeen N, Weinberg AD, Colombo MP. Triggering of OX40 (CD134) on CD4(+)CD25+ T cells blocks their inhibitory activity: a novel regulatory role for OX40 and its comparison with GITR. Blood. 2005;105(7):2845-51.

21. Vu MD, Xiao X, Gao W, Degauque N, Chen M, Kroemer A, et al. OX40 costimulation turns off Foxp3+ Tregs. Blood. 2007;110(7):2501-10.

22. Broz ML, Binnewies M, Boldajipour B, Nelson AE, Pollack JL, Erle DJ, et al. Dissecting the tumor myeloid compartment reveals rare activating antigen-presenting cells critical for T cell immunity. Cancer Cell. 2014;26(5):638-52.

23. Roberts EW, Broz ML, Binnewies M, Headley MB, Nelson AE, Wolf DM, et al. Critical Role for CD103(+)/CD141(+) Dendritic Cells Bearing CCR7 for Tumor Antigen Trafficking and Priming of T Cell Immunity in Melanoma. Cancer Cell. 2016;30(2):324-36.

24. Eisenbarth SC. Dendritic cell subsets in T cell programming: location dictates function. Nat Rev Immunol. 2019;19(2):89-103.

25. Dorner BG, Dorner MB, Zhou X, Opitz C, Mora A, Güttler S, et al. Selective expression of the chemokine receptor XCR1 on cross-presenting dendritic cells determines cooperation with CD8+ T cells. Immunity. 2009;31(5):823-33.

26. Crozat K, Tamoutounour S, Vu Manh TP, Fossum E, Luche H, Ardouin L, et al. Cutting edge: expression of XCR1 defines mouse lymphoid-tissue resident and migratory dendritic cells of the CD8 $\alpha+$ type. J Immunol. 2011;187(9):4411-5.

27. Ahn E, Araki K, Hashimoto M, Li W, Riley JL, Cheung J, et al. Role of PD-1 during effector CD8 T cell differentiation. Proc Natl Acad Sci U S A. 2018;115(18):4749-54.

28. Croft M, So T, Duan W, Soroosh P. The significance of OX40 and OX40L to T-cell biology and immune disease. Immunol Rev. 2009;229(1):173-91.

29. Shevtsov M, Multhoff G. Heat Shock Protein-Peptide and HSP-Based Immunotherapies for the Treatment of Cancer. Front Immunol. 2016;7:171.

30. Obeid M, Tesniere A, Ghiringhelli F, Fimia GM, Apetoh L, Perfettini JL, et al. Calreticulin exposure dictates the immunogenicity of cancer cell death. Nat Med. 2007;13(1):54-61.

31. Mroz P, Vatansever F, Muchowicz A, Hamblin MR. Photodynamic therapy of murine mastocytoma induces specific immune responses against the cancer/testis antigen P1A. Cancer Res. 2013;73(21):6462-70.

32. Mroz P, Szokalska A, Wu MX, Hamblin MR. Photodynamic therapy of tumors can lead to development of systemic antigen-specific immune response. PLoS One. 2010;5(12):e15194.

33. Huang AY, Gulden PH, Woods AS, Thomas MC, Tong CD, Wang W, et al. The immunodominant major histocompatibility complex class I-restricted antigen of a murine colon tumor derives from an endogenous retroviral gene product. Proc Natl Acad Sci U S A. 1996;93(18):9730-5.

34. Hazeldine J, Hampson P, Lord JM. The diagnostic and prognostic value of systems biology research in major traumatic and thermal injury: a review. Burns Trauma. 2016;4:33.

35. Iwai Y, Ishida M, Tanaka Y, Okazaki T, Honjo T, Minato N. Involvement of PD-L1 on tumor cells in the escape from host immune system and tumor immunotherapy by PD-L1 blockade. Proc Natl Acad Sci U S A. 2002;99(19):12293-7.

36. Fanoni D, Tavecchio S, Recalcati S, Balice Y, Venegoni L, Fiorani R, et al. New monoclonal antibodies against B-cell antigens: possible new strategies for diagnosis of primary cutaneous B-cell lymphomas. Immunol Lett. 2011;134(2):157-60.

37. Terme M, Ullrich E, Aymeric L, Meinhardt K, Desbois M, Delahaye N, et al. IL-18 induces PD-1-dependent immunosuppression in cancer. Cancer Res. 2011;71(16):5393-9.

38. Ostrand-Rosenberg S, Horn LA, Haile ST. The programmed death-1 immune-suppressive pathway: barrier to antitumor immunity. J Immunol. 2014;193(8):3835-41.

39. Peng Q, Qiu X, Zhang Z, Zhang S, Zhang Y, Liang Y, et al. PD-L1 on dendritic cells attenuates T cell activation and regulates response to immune checkpoint blockade. Nat Commun. 2020;11(1):4835.

40. Zou W, Chen L. Inhibitory B7-family molecules in the tumour microenvironment. Nat Rev Immunol. 2008;8(6):467-77. 
41. Dong H, Strome SE, Salomao DR, Tamura H, Hirano F, Flies DB, et al. Tumor-associated B7-H1 promotes Tcell apoptosis: a potential mechanism of immune evasion. Nat Med. 2002;8(8):793-800.

42. Konishi J, Yamazaki K, Azuma M, Kinoshita I, Dosaka-Akita H, Nishimura M. B7-H1 expression on non-small cell lung cancer cells and its relationship with tumor-infiltrating lymphocytes and their PD-1 expression. Clin Cancer Res. 2004;10(15):5094-100.

43. Pan PY, Zang Y, Weber K, Meseck ML, Chen SH. OX40 ligation enhances primary and memory cytotoxic T lymphocyte responses in an immunotherapy for hepatic colon metastases. Mol Ther. 2002;6(4):528-36.

44. Linch SN, McNamara MJ, Redmond WL. OX40 Agonists and Combination Immunotherapy: Putting the Pedal to the Metal. Front Oncol. 2015;5:34.

45. Krysko DV, Garg AD, Kaczmarek A, Krysko O, Agostinis P, Vandenabeele P. Immunogenic cell death and DAMPs in cancer therapy. Nat Rev Cancer. 2012;12(12):860-75.

46. Zelenay S, Reis e Sousa C. Adaptive immunity after cell death. Trends Immunol. 2013;34(7):329-35.

47. Loke P, Allison JP. PD-L1 and PD-L2 are differentially regulated by Th1 and Th2 cells. Proc Natl Acad Sci U S A. 2003;100(9):5336-41.

48. Topalian SL, Drake CG, Pardoll DM. Targeting the PD-1/B7-H1(PD-L1) pathway to activate anti-tumor immunity. Curr Opin Immunol. 2012;24(2):207-12.

49. Eppihimer MJ, Gunn J, Freeman GJ, Greenfield EA, Chernova T, Erickson J, et al. Expression and regulation of the PD-L1 immunoinhibitory molecule on microvascular endothelial cells. Microcirculation. 2002;9(2):133-45.

50. Keir ME, Butte MJ, Freeman GJ, Sharpe AH. PD-1 and its ligands in tolerance and immunity. Annu Rev Immunol. 2008;26:677-704.

51. Latchman Y, Wood CR, Chernova T, Chaudhary D, Borde M, Chernova I, et al. PD-L2 is a second ligand for PD-1 and inhibits T cell activation. Nat Immunol. 2001;2(3):261-8.

52. Frigola $\mathrm{X}$, Inman BA, Lohse $\mathrm{CM}, \mathrm{Krco} \mathrm{CJ}$, Cheville JC, Thompson RH, et al. Identification of a soluble form of B7-H1 that retains immunosuppressive activity and is associated with aggressive renal cell carcinoma. Clin Cancer Res. 2011;17(7):1915-23.

53. Vinay DS, Ryan EP, Pawelec G, Talib WH, Stagg J, Elkord E, et al. Immune evasion in cancer: Mechanistic basis and therapeutic strategies. Semin Cancer Biol. 2015;35 Suppl:S185-s98.

54. Spranger S. Mechanisms of tumor escape in the context of the T-cell-inflamed and the non-T-cell-inflamed tumor microenvironment. Int Immunol. 2016;28(8):383-91.

30

31

32 


\section{Figure and Tables}
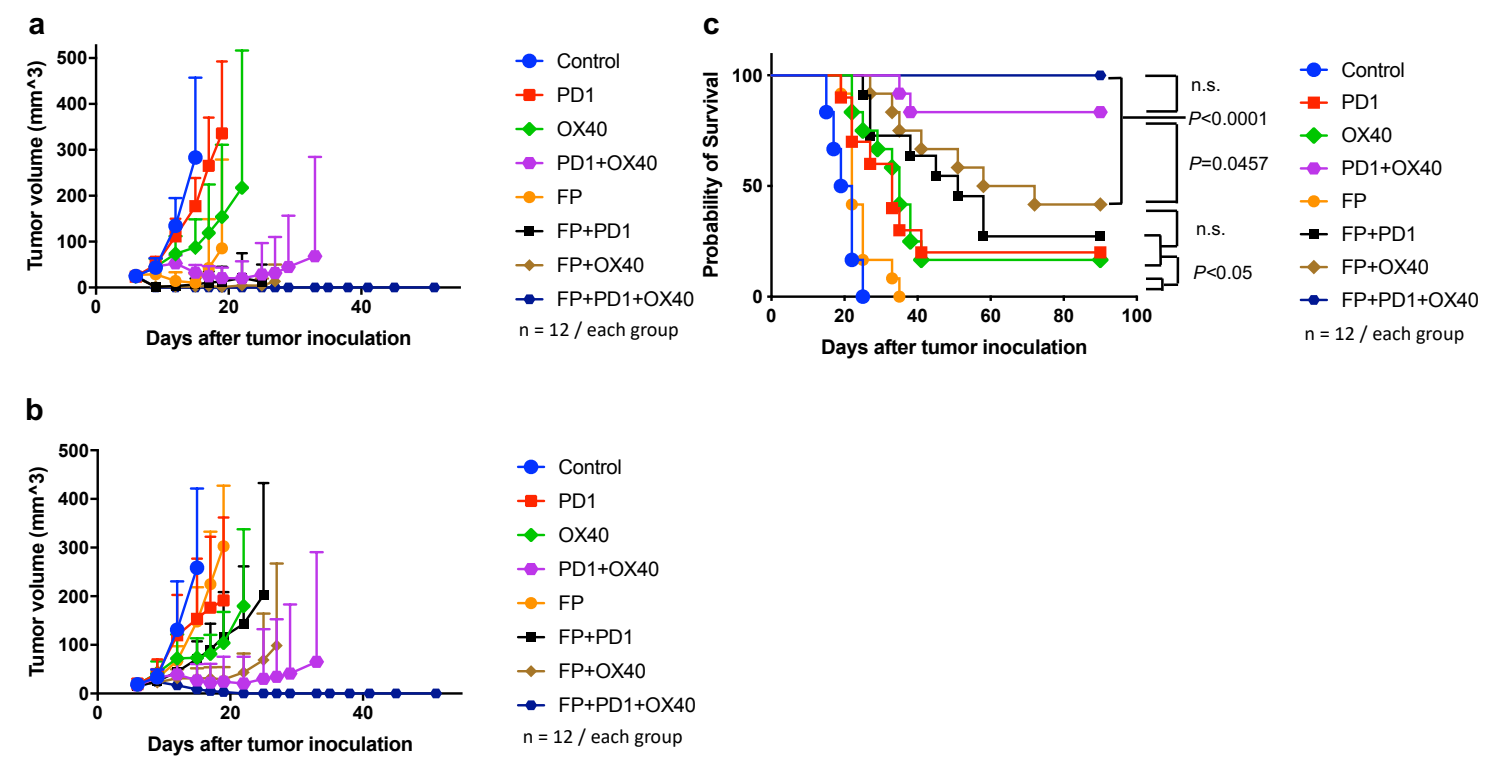

Fig. 1

Figure 1. 6-day inoculation tumor volume and survival curves post treatment.

5 To investigate whether aFP treatment can induce systemic anti-tumor immunity, we established a

6 mouse model with tumors on both hind legs. Tumors on the left were treated with aFP and

7 growth of both tumors was observed. aFP laser irradiation was performed 6 days after tumor

8 inoculation. Anti-PD-1 inhibitor and OX40 agonist were administered intraperitoneally at a dose

9 of $200 \mu \mathrm{g}$ per mouse on days $6,8,10,12$ and 14 after tumor cell inoculation.

(a) Average tumor volume curves on the aFP-treated legs of mice treated on day 6.

(b) Average tumor volume curves on the untreated contralateral legs of mice treated on day 6. 
1 (c) Kaplan-Meier survival curves of mice receiving tumor inoculation, aFP-treated on day 6. The

2 significance values for the difference between the survival curves are shown in Table 1.

3 : Control vs. atni-PD-1: $P=0.0007$, Control vs. OX40: $P<0.0001$, Control vs. Anti-PD-1 +

4 OX40: $P<0.0001$, Control vs. aFP: $P=0.0217$, Control vs. aFP + anti-PD-1: $P<0.0001$,

5 Control vs. aFP + OX40: $P<0.0001$, Control vs. aFP + anti-PD-1 + OX40: $P<0.0001$, anti-

6 PD-1 vs. OX40: n.s., anti-PD-1 vs. Anti-PD-1 + OX40: $P=0.001$, anti-PD-1 vs. aFP: $P=$

7 0.0281, anti-PD-1 vs. aFP + anti-PD-1: n.s., anti-PD-1 vs. aFP + OX40: n.s., anti-PD-1 vs. aFP +

8 anti-PD-1 + OX40: $P<0.0001$, OX40 vs. Anti-PD-1 + OX40: $P=0.0007$, OX40 vs. aFP: $P=$

$9 \quad 0.0018$, OX40 vs. aFP + anti-PD-1: n.s., OX40 vs. aFP + OX40: n.s., OX40 vs. aFP + anti-PD-1

$10+$ OX40: $P<0.0001$, Anti-PD-1 + OX40 vs. aFP: $P<0.0001$, Anti-PD-1 + OX40 vs. aFP + anti-

11 PD-1: $P=0.01$, Anti-PD-1 + OX40 vs. aFP + OX40: $P=0.0457$, Anti-PD-1 + OX40 vs. aFP +

12 anti-PD-1 + OX40: n.s., aFP vs. aFP + anti-PD-1: $P<0.0001$, aFP vs. aFP + OX40: $P<0.0001$,

13 aFP vs. aFP + anti-PD-1 + OX40: $P<0.0001$, aFP + anti-PD-1 vs. aFP + OX40: n.s., aFP + anti-

14 PD-1 vs. aFP + anti-PD-1 + OX40: $P=0.0002$, aFP + OX40 vs. aFP + anti-PD-1 + OX40: $P<$

150.0001. 
a

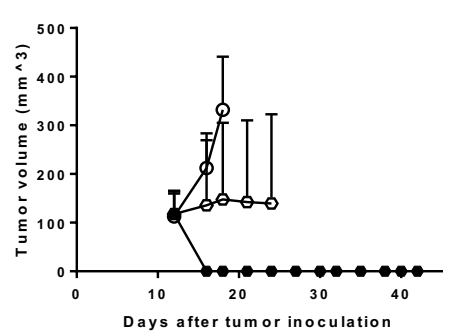

b

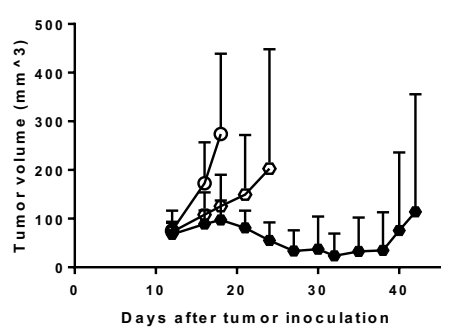

c

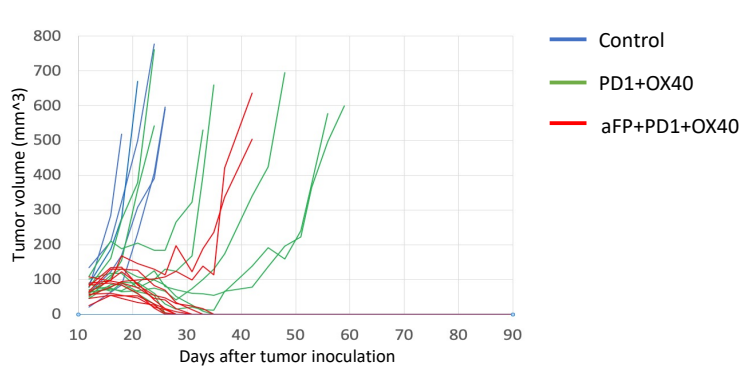

d

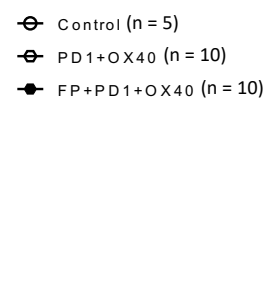

$\bigoplus$ Control $(n=5)$

$\rightarrow$ PD $1+0 \times 40(n=10)$

$\rightarrow F P+P D 1+O \times 40(n=10)$

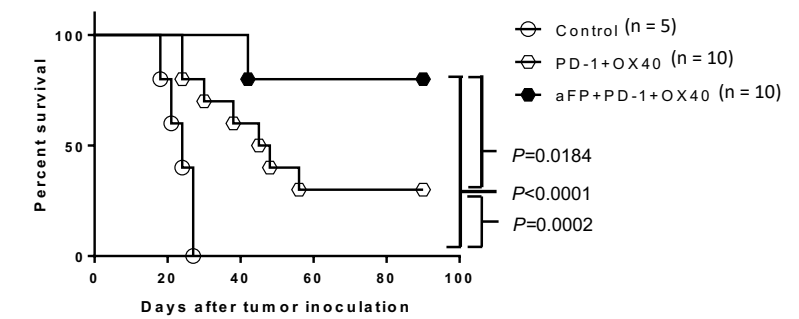

\section{Figure 2. 12-day inoculation tumor volume and survival curves post treatment.}

3 To investigate the relative efficacy of anti-PD-1 + OX40 agonist therapy vs triple therapy (aFP +

4 anti-PD-1 + OX40 agonist) in large tumors, aFP and immunomodulatory treatment was started

512 days after tumor inoculation. Anti-PD-1 inhibitor and OX40 agonist were administered

6 intraperitoneally at a dose of $200 \mu \mathrm{g}$ per mouse on days $12,14,16,18$ and 20 after tumor cell

7 inoculation.

8 (a) Average tumor volume curves in tumors treated with aFP on day 12.

9 (b) Average tumor volume curves on the untreated contralateral tumors in mice treated with aFP 10 on day 12 .

11 (c) Individual tumor volume curves on the untreated contralateral tumor in mice treated with aFP

12 on day 12. 
1 (d) Kaplan-Meier survival curves in mice receiving aFP treatment 12 days post tumor

2 inoculation. Significance values between survival curves are: Control vs. Anti-PD-1 + OX40: $P$

$3=0.0022$, Control vs. aFP + anti-PD-1 + OX40: $P<0.0001$, Anti-PD-1 + OX40 vs. aFP + anti-

$4 \quad$ PD-1 + OX40: $P=0.0252$.

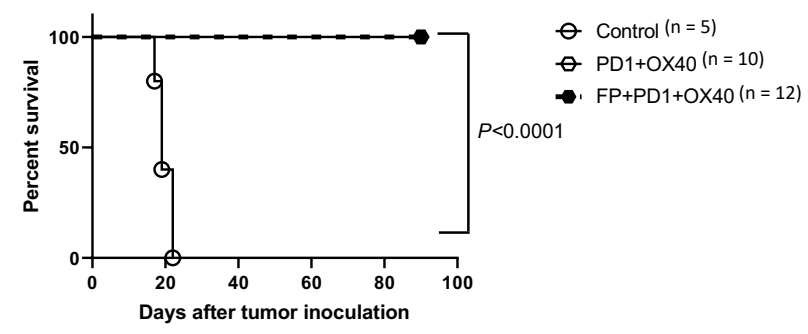

\section{$7 \quad$ Figure 3. Survival curves on rechallenge.}

8 Kaplan-Meier survival curves of mice receiving the rechallenge test with CT26WT cells. Each

9 mouse was inoculated subcutaneously with $3.5 \times 10^{5}$ CT26WT cells on the aFP-untreated

10 contralateral thigh. Significance values between survival curves are: control mice vs. survival

11 mice in anti-PD-1 + OX40 and aFP + anti-PD-1 + OX40: $P<0.0001$. 
a


b

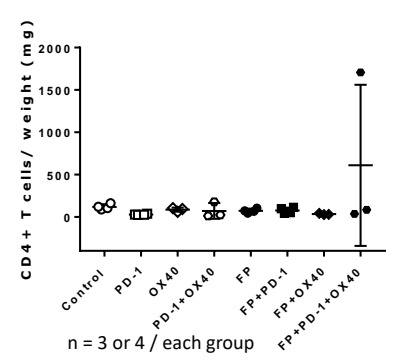

e

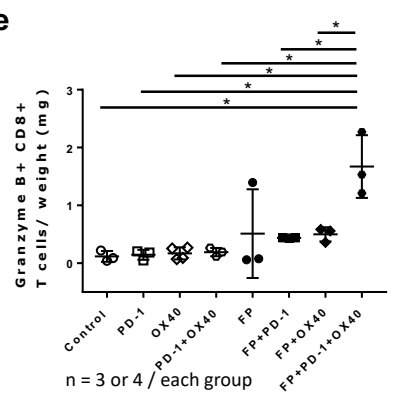

C

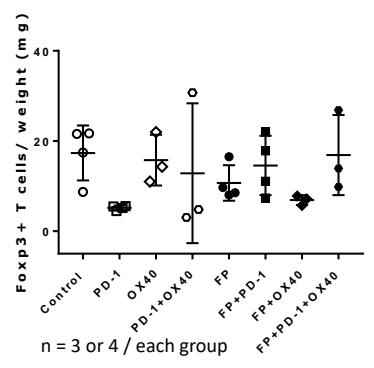

f

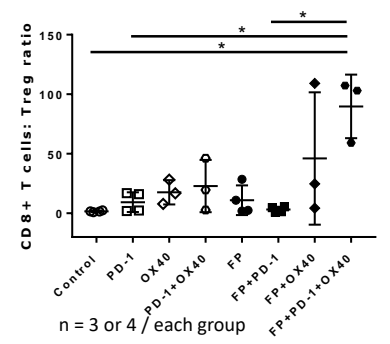

Figure 4. Flow cytometric analysis of tumor infiltrating lymphocytes 5 days post aFP treatment in untreated contralateral tumors.

4 Flow cytometry analysis was performed 5 days after aFP treatment to investigate the number of

$5 \mathrm{CD} 3+, \mathrm{CD} 4+$, and $\mathrm{CD} 8+$, and granzyme $\mathrm{B}+\mathrm{T}$-cells in addition to the ratio of $\mathrm{T}_{\text {regs }}$ expressing

$6 \mathrm{CD} 4$ and Foxp3 in the untreated contralateral tumor.

7 (a) Proportion of CD3+ T-cells cells normalized to tumor weight.

8 (b) Proportion of CD4+ T-cells normalized to tumor weight.

9 (c) Proportion of Treg normalized to tumor weight.

(d) Proportion of CD8+ T cells normalized to tumor weight.

(e) Proportion of granzyme $\mathrm{B}+\mathrm{CD} 8+\mathrm{T}$ cells normalized to tumor weight.

(f) Ratio of CD8+ T cells to Tregs (CD4+Foxp3+). 
a

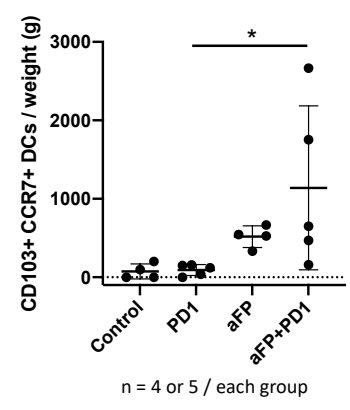

b

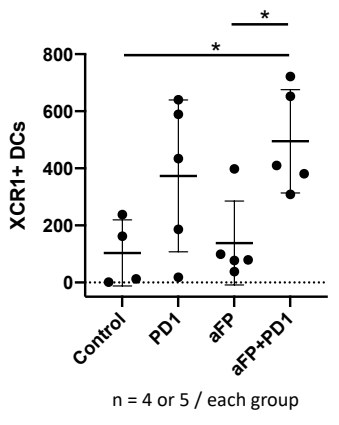

2 Figure 5. Flow cytometric analysis of dendritic cells (DCs) 3 days post aFP treatment in 3 aFP-treated tumor and local drainage lymph node.

4 Flow cytometry analysis was performed 3 days after aFP treatment to investigate the number of

5 CD103+ CCR7+ DCs in aFP-treated tumor and XCR1+ DCs in drainage lymph node on the 6 treated side.

7 (a) Proportion of CD103+ CCR7+ DCs normalized to tumor weight.

(b) Absolute number of XCR1+ DCs in drainage lymph node. 

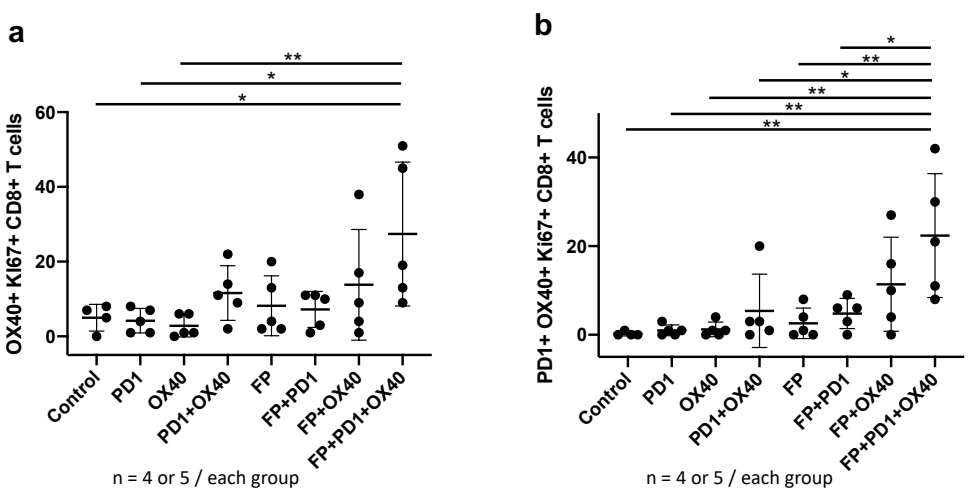

2 Figure 6. Flow cytometric analysis for $\mathrm{OX} 40+\mathrm{Ki67}+\mathrm{CD8}+\mathrm{T}$ cells 5 days after aFP treatment in local drainage lymph node.

4 Flow cytometric analysis was performed 5 days post aFP treatment to quantify the number of

$5 \mathrm{OX} 40+\mathrm{Ki} 67+\mathrm{CD} 8+\mathrm{T}$-cells in drainage lymph node on the aFP-treated side.

6 (a) Absolute number of OX40+ Ki67+ CD8+ T-cells in drainage lymph node on aFP-treated side.

7 (b) Absolute number of PD-1+ OX40+ Ki67+ CD8+ T-cells in drainage lymph node on aFP-treated 8 side. 
a

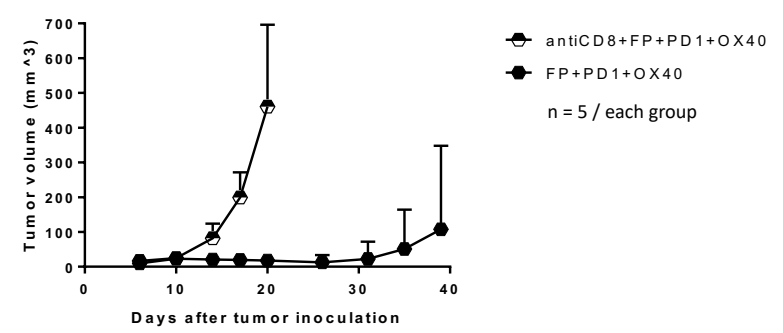

b

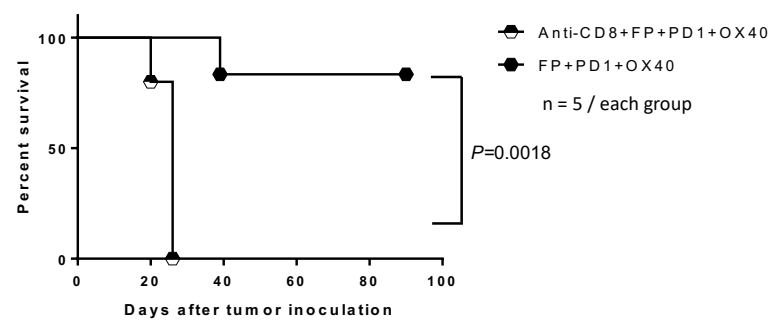

\section{Figure 7. Tumor volume and survival curves after triple therapy with anti-CD8 depletion}

3 antibodies.

4 To investigate whether adaptive immunity is necessary to eradicate cancer cells after the triple

5 therapy, tumor inoculation was performed with an anti-CD8 depletion antibody to wipe out

$6 \mathrm{CD} 8+\mathrm{T}$-cells in the mouse tumor model. Anti-CD8 depletion antibodies were administered

7 intraperitoneally at a dose of $200 \mu \mathrm{g}$ every 3 days from the day before tumor inoculation to

8 removal of mice as an endpoint. (a) Difference in tumor volume over time between experimental

9 groups. (b) Kaplan-Meier survival curves showing differences in mortality in mice receiving

10 triple therapy $+/$ - anti-CD8 depletion antibody. The significance values for the difference

11 between the survival curves are: anti-CD8+aFP vs. aFP + anti-PD-1 + OX40: $P=0.0018$. 
a

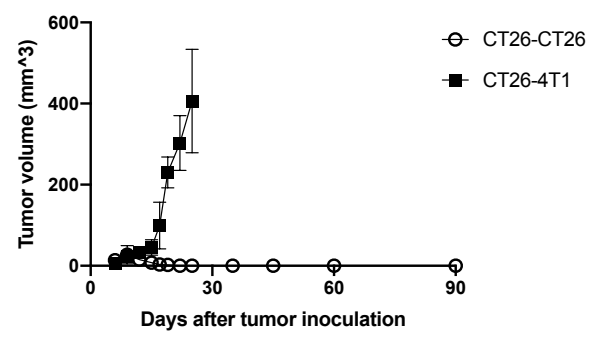

b

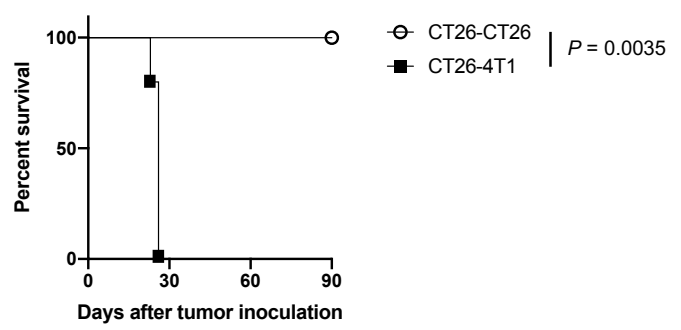

2 Figure 8. Tumor volume and survival curves after treatment in a mismatched CT26WT and 4T1 tumor model.

4 To determine whether the observed shrinkage of the contralateral, untreated tumor in triple therapy is due

5 to antigen specific immunity induced by aFP, we compared outcomes in matched and mismatched tumor

6 models. In the matched group, mice were inoculated with CT26WT in both legs. In the mismatched

7 group, mice were injected with CT26WT tumor cells in the left leg and 4T1 (murine mammary

8 carcinoma) cells in the right leg. Both groups were treated with triple therapy and aFP was performed on

9 the left CT26WT leg alone. (a) Volume of the right untreated contralateral CT26WT or 4T1 tumor in

10 triple therapy. (b) Kaplan-Meier curves in matched and mismatched tumor model receiving triple therapy.

11 Bars represent SD. The significance values for the difference between the survival curves are: CT26-

12 CT26 vs. CT26-4T1:P=0.0035. 


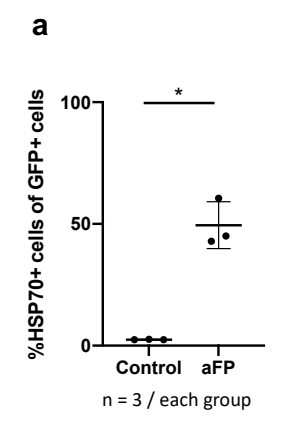

b

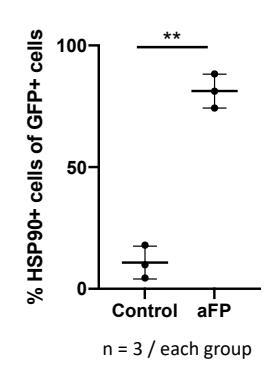

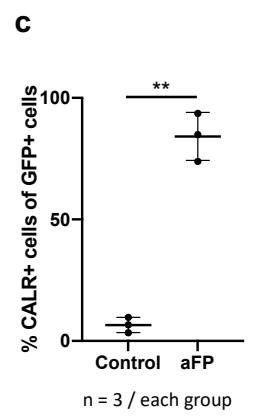

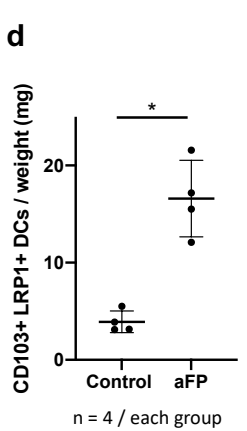

1

2 Figure 9. Flow cytometric analysis of DAMPs-expressing tumor cells and DCs expressing

$3 \quad$ LRP1 3 days after aFP treatment in aFP-treated tumors.

4 Flow cytometry analysis was performed 3 days after aFP treatment to investigate percentage of

5 HSP70, HSP90 and CALR-expressing GFP+ tumor cells and the number of LRP1+ DCs in aFP-

6 treated tumors.

7 (a) Percentage of GFP+ cells expressing HSP70.

8 (b) Percentage of GFP+ cells expressing HSP90.

9 (c) Percentage of GFP+ cells expressing CALR.

10

(d) Concentration of LRP1+ DCs normalized to tumor weight. 

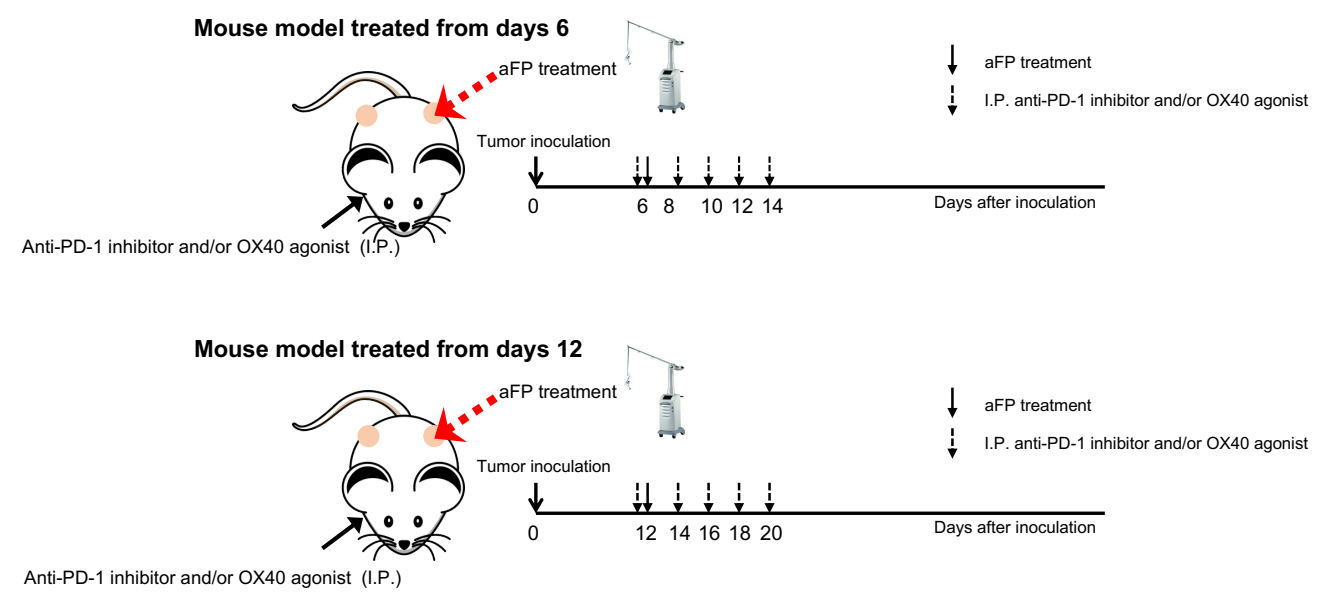
a

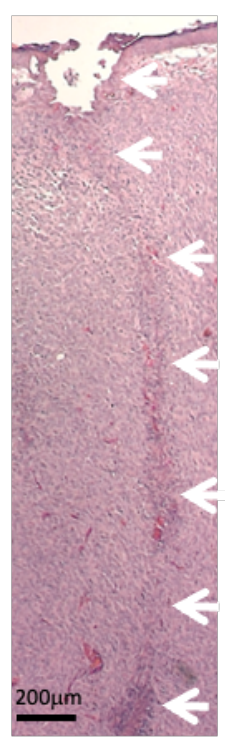

b

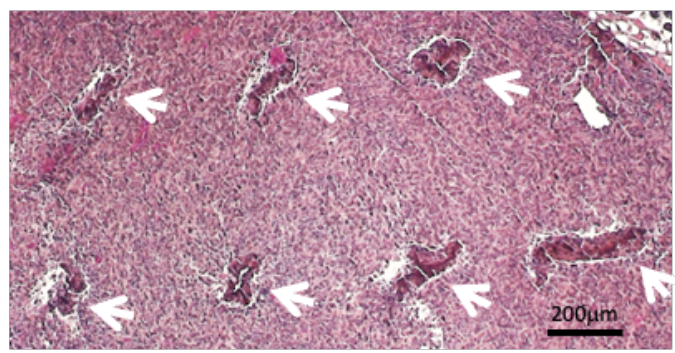

Supplement figure 1

\section{Supplementary figure 1.}

3 H \& E stained histologic figures of tumor tissue harvested immediately after ablative fractional

4 photothermolysis (aFP) laser treatment with a pulse energy of $100 \mathrm{~mJ}$ at a nominal density of

$55 \%$. (a) Parallel view to trajectory of laser beam (b) Orthogonal view to trajectory of laser beam.

6 White arrows indicate an ablated hole characteristic of aFP procedures. The ablated hole

7 appeared to be collapsed and distorted within the tumor tissue. 




\section{Supplementary figure 2.}

4 To investigate the up-regulation of PD-L1 receptors in tumors after aFP treatment, Green

5 fluorescence Protein (GFP) gene was transfected to CT26WT cells using a lentivirus vector

6 (GenTarget Inc). Both aFP-irradiated and non-irradiated tumors were harvested 3 days after

7 irradiation. For isolation of GFP+CT26WT cells, fresh tumors were dissociated mechanically filtering

8 through $70 \mu \mathrm{m}$ strainer on 6 well culture plate with DNaseI $(10 \mu \mathrm{g} / \mathrm{ml}$, Roche; Nutley, NJ) and

9 collagenase $(10 \mathrm{mg} / \mathrm{ml}$, Life technologies $)$ and incubated 60 minutes at $37^{\circ} \mathrm{C}$. The dissociated cells were

10 stained with anti- PD-L1(MIH7, BioLegend; San Diego; CA)) for 30 minutes at $4^{\circ} \mathrm{C}$. Following staining

11 for surface markers, cells were fixed by Foxp3/ Transcription Factor Staining Buffer Set (eBioscience;

12 Santa Clare, CA) according to the manufacturer's instructions at room temperature for 30 minutes. After

13 fixation cells were suspended with washing buffer at $4^{\circ} \mathrm{C}$ over night. The next day the stained cells were 14 analyzed on Fortessa X-20 (BD Biosciences; San Jose, CA).

15 
Data of RNA sequence (aFP vs control group)

\begin{tabular}{|c|c|c|c|}
\hline Symbol & log2FoldChange(aFP/Control) & Probability & Up/Down-Regulation(aFP/Control) \\
\hline Hspa1a & 7.725630695 & 1 & Up \\
Hspa1b & 8.309049618 & 0.99999999 & Up \\
II1a & 3.746162754 & 0.99253465 & Up \\
II1b & 3.446302695 & 0.999999807 & Up \\
Ifnb1 & 4.009460329 & 0.987913968 & Up \\
\hline
\end{tabular}

\section{Supplementary table 1. Data of RNA Sequence (aFP vs control group)}

3 To investigate the up-regulation of IL-1 and IFN-beta at the RNA level, we measured RNA

4 expression of IL-1 and IFN-beta in tumors using RNA sequence analysis. aFP-irradiated tumor

5 and non-irradiated tumor were harvested 24 hours after irradiation. RNA was extracted using

6 RNeasy mini kit (Qiagen) and RNA sequencing was performed by a commercial company

7 (Beijing Genomics Institute). Each group contain 3 tumors. The table shows aFP-treatment

8 induces IL-1 and IFN-beta expression in tumor cells. 